\title{
Title: Electron microscopy of electromagnetic waveforms
}

\author{
Authors: A. Ryabov ${ }^{1,2}$ and P. Baum ${ }^{1,2 *}$
}

\begin{abstract}
Affiliations:
${ }^{1}$ Ludwig-Maximilians-Universität München, Am Coulombwall 1, 85748 Garching, Germany

${ }^{2}$ Max-Planck-Institute of Quantum Optics, Hans-Kopfermann-Str. 1, 85748 Garching, Germany

*Correspondence to: peter.baum@1mu.de
\end{abstract}

\begin{abstract}
Rapidly changing electromagnetic fields are the basis of almost any photonic or electronic device operation. We report how electron microscopy can measure collective carrier motion and fields with sub-cycle and sub-wavelength resolution. A collimated beam of femtosecond electron pulses passes through a metamaterial resonator that is previously excited with a single-cycle electromagnetic pulse. If the probing electrons are shorter in duration than half a field cycle, then time-frozen Lorenz forces distort the images quasi-classically and with sub-cycle time resolution. A pump-probe sequence reveals a movie of the sample's oscillating electromagnetic field vectors with time, phase, amplitude, and polarization information. This waveform electron microscopy can be used to visualize electrodynamic phenomena in devices as small and fast as available.
\end{abstract}

One-sentence summary: Electron microscopy images electromagnetic field oscillations with sub-cycle and sub-wavelength resolution. 
Main Text: Electron microscopy works at 100,000-fold smaller wavelengths than light and therefore allows studying matter and materials with sub-atomic resolution $(1,2)$. With added temporal resolution, ultrafast reaction paths in physical and chemical transitions can also be recorded $(3,4)$.

Rather elusive for electron microscopy, however, have been electrodynamic phenomena, although oscillating currents and fields are fundamental to the operation of almost any information processing device or metamaterial. Based on differential phase contrast $(5,6)$, ptychography (7) or laser-electron energy exchange techniques (8), electron microscopy studies on electromagnetism could reveal electrostatic field distributions (9-11), ultrafast carrier diffusions $(12,13)$ or cycle-averaged nanophotonic dynamics $(14-16)$, but not so far the fundamental electromagnetic waveforms with their rapidly oscillating field vectors.

We merge the electron microscope's supremacy in matter characterizations with a subcycle and sub-wavelength access to electromagnetic phenomena (Fig. 1). Femtosecond electron pulses (blue) at $70 \mathrm{keV}$ central energy are generated by pulsed laser photoemission (17). The electron wavepackets (18) are further compressed in time by a THz field (red) in grazing incidence to a foil (19). Alternatives here could be beam blanking (20), microwave compression (18), photon-gating (21) or ponderomotive bunching (22). A magnetic lens (grey) widens the beam for passage through the sample with close-to-zero divergence. The proof-of-principle sample is a metal split-ring resonator (yellow), which is a typical building block for metamaterials (23) or surfaces (24) with optical effects otherwise not available $(25,26)$. The resonator with $\sim 250 \mu \mathrm{m}$ radius is excited with a single-cycle, phase-locked electromagnetic pulse of 0.1-0.8 THz bandwidth (17) propagating along $z$ with a linear polarization oriented $\sim 5^{\circ}$ off the $y$ axis. The electron pulse duration at the sample is $\sim 15$ times shorter than the excitation halfcycle. An objective magnetic lens (grey) magnifies the shadowed electron beam onto a screen (green) with some intentional defocus. This makes the scheme sensitive to local beam deflections (dotted) and allows concluding from the distorted screen images, taken at a sequence of electron/field delay times, to the time-frozen electrodynamics in the sample. 
First results are shown in Fig. 2, obtained with the excitation field depicted in Fig. 2A and at a magnification of $\sim 5$. The electron pulses are characterized by streaking (19) and have 80 -fs duration (Fig. 2B). The resonator, which was laser-machined into $30-\mu \mathrm{m}$ thick aluminum foil (Fig. 2C), shows some imperfections, particularly fringy edges and off-center circles. An isosurface of the time-dependent shadow pattern deformations reveals pronounced temporal oscillations (Fig. 2D). In the raw image details for a subset of pump-probe delays (Fig. 2E), the shadow pattern at $-\infty$ becomes distorted in a self-overlapping way before returning to the original at later times; see movie S1. Another example of raw data is shown in movie S2.

For analyzing our experiment, we denote the spatiotemporal electric and magnetic fields around the resonator with $\boldsymbol{E}(x, y, z, t)$ and $\boldsymbol{B}(x, y, z, t) ; v_{e l} \approx 0.526 c$ (speed of light) is the electron velocity, $\tau_{e l} \approx 80$ fs the electron pulse duration, $f_{\max } \approx 0.8 \mathrm{THz}$ the highest frequency excited, $D_{z} \approx 30 \mu \mathrm{m}$ the mode thickness along $z$ and $\alpha_{e m} \approx 60 \mu \mathrm{rad}(\mathrm{rms})$ the beam divergence at the sample ( $\sim 20 \mathrm{~nm}$ source emittance and $\sim 340 \mu$ m beam radius). We make these approximations: (a) $\tau_{e l} \ll 1 / f_{\max }$, i.e. the electron pulses are much shorter than a wave cycle. (b) $D_{z} / v_{e}<<1 / f_{\text {max }}$, i.e. sub-cycle transition time. (c) $\alpha_{e m} \approx 0$, i.e. the probe beam is collimated. (d) Two-dimensional fields $E_{x, y}(x, y, t)$ and $B_{x, y}(x, y, t)$ suitably describe the resonator response. (e) Magnetic fields are negligible (17). Note that (a)-(b) are essential to the concept and (c)-(e) specific to the particular geometry. With $m_{e}$ and $e$ the electron mass and charge, respectively, we obtain for the change in angles $\alpha_{x, y}$ at each position in the beam, at each pump-probe delay $\tau$ :

$$
\alpha_{x, y}(x, y, \tau) \approx \frac{e E_{x, y}(x, y, \tau) D_{z}}{m_{e} v_{e}^{2}} .
$$

Each ray in the electron beam profile acquires a local-field-dependent momentum kick that is directly proportional to the local waveform, frozen in time at the chosen probe delay.

On the screen, electrons from faraway locations can end up at the same positions and inversion is non-bijective. We therefore measure for each pump-probe delay a batch of screen images, in which the excitation peak field strength $E_{\text {exc }}$ is gradually increased from zero to the available maximum. Due to the collimated illumination, this method is related to measuring the evolution of the distorted beam along the $z$-axis. The dataset is four-dimensional and comprises 
$250 \times 250$ image pixels, 150 pump-probe delays and 16 different excitation field strengths. At $50 \mathrm{kHz}$ pump-probe repetition rate, the total accumulation time is $\sim 1$ hour. We calculate $\alpha_{x, y}(x, y, \tau)$ for each delay step with a least-square fitting algorithm $(17)$ and $E_{x, y}(x, y, \tau)$ via Eq. (1). Figure 3A shows from the full results (movie S3) three vector-field snapshots at 2.8, 3.2 and 3.8 ps delay, respectively; the triangle tips denote the vectorial direction and field strength is encoded in color and size. Some high-frequency temporal noise was diminished with a low-pass Gaussian filter at $1 \mathrm{THz}$. The vector field at 2.8 ps shows three local maxima (top, right and left). We see an asymmetry in $x$, a predominantly radial polarization everywhere, and at each angle a radially decreasing field strength. The peak field is $7 \mathrm{~V} / \mu \mathrm{m}$, which is $\sim 3.5$ times higher than the driving field. Figure 3B shows at two selected positions (white circles in Fig. 3A) the timedependent electric fields. Time-dependent polarization is plotted in the two right panels. The fields initially follow the single-cycle excitation pulse, but after $\sim 4 \mathrm{ps,}$, where the excitation has diminished, they evolve into slower oscillation cycles with decaying amplitude. Fitting each such time trace with a damped-harmonic oscillator model reveals a map of central frequencies $(0.27-$ $0.30 \mathrm{THz})$, dampings $(3-6 \mathrm{ps})$ and group delays $( \pm 0.1 \mathrm{ps})$. These ranges indicate that a single delocalized mode is predominant after the excitation.

Figure 3C shows an analysis of the collective carrier motion causing the observed nearfield dynamics. The surface charge density $\sigma=\varepsilon_{0} E_{\perp}$ is plotted along a path around the inner edge of the resonator. The peak charge is $\sim 400 e / \mu \mathrm{m}^{2}$ and $\sim 20 e / \mu \mathrm{m}^{2}$ are detectable above the noise. Three spatial regions are evident, one at the top $\left( \pm 180^{\circ}\right)$ and two others left and right of the gap $\left(\sim \pm 45^{\circ}\right)$. The dynamics around the gap extends by more than \pm 90 and is phase-shifted with respect to the top region. Some features in Fig. 3C have a tilt, indicating in part an azimuthally traveling excitation, which is also observable in the raw data and result movies ( $\mathrm{S} 1$ and S3, respectively). Circular motion ceases after $\sim 4$ ps and the remaining dynamics is mostly symmetric with $x$. It appears that the excitation first localizes at the gap and top half, while subsequently creating a spread-out, rather symmetric and longer-lived mode with mostly radial polarization. 
Next, we used our waveform electron microscopy to characterize a rectangular aperture and butterfly resonator, typical elements for field enhancement in optics. Figure 4A shows a snapshot at $2.2 \mathrm{ps}$ delay of the field in the slit $\left(730 \times 100 \mu \mathrm{m}^{2}\right)$; see also movie S4. Figure 4B shows the waveform in the center. The frequency at early times is higher than later, where the oscillations show a non-harmonic shape with damped peak regions. This indicates the presence of multiple modes at different frequencies, but phase-locked. In order to find those, we invoke a principle component analysis via singular value decomposition. Figure 4C depicts the first four modes; further ones have no clear shape or time structure anymore. Three of the modes have a series of maxima that are almost equidistantly distributed over the slit length. Mode $1 \mathrm{~b}$ is different and has a shape with vortex polarization; we attribute this to residual magnetic field effects (17) and a slight tilt of the structure with respect to the electron beam. Figure 4D shows the time traces from the decomposition matrix. Mode 1 is centered at $0.20 \mathrm{THz}$, mode 2 at $0.39 \mathrm{THz}$ and mode 3 at $0.54 \mathrm{THz}$. Figure 4E shows cuts through the modes along the slit's long axis at center. Evident are one, two and three spatial maxima, respectively, but with some asymmetries and slightly elliptical polarization in modes 2 and 3. Note that singular value decomposition neither assumes any electromagnetic boundary conditions nor enforces harmonic-oscillator solutions. That nevertheless a series of oscillation orders with dispersion and nontrivial phase relations can be identified, is a virtue of the complete spatial, temporal and vectorial dataset obtained with waveform electron microscopy. In the butterfly-shaped resonator (Fig. 4F and movie S5), there is only one mode excited (frequency $0.3 \mathrm{THz}$, damping $\sim 5 \mathrm{ps}$ ). The field enhancement at $2.8 \mathrm{ps}$ is $\sim 9$ close to the metal and $\sim 6$ in the middle, which is lower than desirable, because the rounded crests of our structure apparently disperse the field lines at the center.

The ability to record field vectors in space and time is essentially a consequence of the electron charge, which is usually detrimental in ultrafast microscopy via space charge effects, but here exploited as a local, sensitive, directional and non-invasive probe of electromagnetic field cycles. We estimate the angular and spatial resolutions achievable in a state-of-the-art instrument at few-nanometer resolution by invoking a ray-optical analysis (17). The result is plotted in Fig. S4 in dependence on defocus. Letting the spatial resolution degrade by only a factor of two is 
already sufficient for an angular resolution below $20 \mu \mathrm{rad}$. Via Eq. (1) and assuming a 100-nm deep structure along $z$, we obtain a measurable field strength of $\sim 0.0026 \mathrm{~V} / \mathrm{nm}$ or $\sim 1 \mathrm{~V}$ per $40 \mathrm{~nm}$, which overlaps with the conditions in working electronic or plasmonic circuitry. In the experiment, the angular resolution is $60-80 \mu \mathrm{rad}(17)$, which is close to $\alpha_{e m} \approx 60 \mu \mathrm{rad}$. At reasonable signal-to-noise levels, the illumination's uncorrelated divergence determines the angular resolution. The ultimate limit with coherent femtosecond emitters (27) and a wave-based analysis $(6,7)$ is defined by the de Broglie wavelength of the electrons.

Magnetic fields, which are important in negative-index metamaterials or memory devices, can be revealed and separated from the electric-field deflections by making two experiments at different electron velocities, because the electric parts of the Lorentz force scales proportionally to $1 / v_{e}{ }^{2}$, while the magnetic deflection follows a $1 / v_{e}$ law. Energy-filtered imaging can measure forward momentum changes and in combination with tomography reveal the complete electric and magnetic vector fields in space and time. Surface studies with a scanning electron microscope are also feasible, given its sensitivity to (transient) surface voltages, energy losses or secondary electron trajectory asymmetries.

Microwave-compressed (18), photon-gated (21) or deflection-selected (20) electron pulses are approaching the few-femtosecond regime of pulse duration. This allows waveform imaging at multi-THz frequencies, faster than any electronic devices available. Metamaterials operate at lightwave frequencies, where $\sim 1$-fs resolution is required for sub-cycle sampling. Recently, attosecond electron pulse trains (28) have been realized in an electron microscope (29). With cycle-synchronous excitation and probing (28), waveform electron microscopy will enable a direct visualization of nanophotonic phenomena at lightwave frequencies. Isolated attosecond electron pulses may potentially be generated via single-electron wavepacket compression (18) or ponderomotive effects (22), and will be useful for studying nonlinear or strong-field phenomena that are not reversible between adjacent excitation cycles.

In principle, any light-matter interaction starts with atomic-scale charge displacements, and waveform electron microscopy at sub-atomic resolution $(5,6)$ could potentially reveal those motions. More straightforward, however, will be studies of collective carrier dynamics and field 
effects in nanoscale devices, for example in electronics, metamaterials, nanophotonic circuitry, near-field sensors or light-harvesting nanostructures. The fact that waveform imaging and shape characterization now require only one instrument, the electron microscope, will probably be advantageous for such investigations. 


\section{References and Notes:}

(1) P. E. Batson, N. Dellby, O. L. Krivanek, Nature 418, 617 (2002).

(2) R. Erni, M. D. Rossell, C. Kisielowski, U. Dahmen, Phys. Rev. Lett. 102, 096101 (2009).

(3) A. H. Zewail, Science 328, 187 (2010).

(4) R. J. D. Miller, Science 343, 1108 (2014).

(5) N. Shibata, et al., Nat Phys 8, 611 (2012).

(6) K. Mueller, et al., Nature Communications 5, 5653 (2014).

(7) M. J. Humphry, B. Kraus, A. C. Hurst, A. M. Maiden, J. M. Rodenburg, Nature Communications 3, 730 (2012).

(8) B. Barwick, D. J. Flannigan, A. H. Zewail, Nature 462, 902 (2009).

(9) J. Cumings, A. Zettl, M. R. McCartney, J. C. H. Spence, Physical Review Letters 88, $056804(2002)$.

(10) M. Lohr, et al., Ultramicroscopy 117, 7 (2012).

(11) N. Shibata, et al., Scientific Reports 5, 10040 (2015).

(12) M. Mueller, A. Paarmann, R. Ernstorfer, Nature Communications 5, 5292 (2014).

(13) E. Najafi, T. D. Scarborough, J. Tang, A. Zewail, Science 347, 164 (2015).

(14) A. Yurtsever, R. M. van der Veen, A. H. Zewail, Science 335, 59 (2012).

(15) E. Quinonez, J. Handali, B. Barwick, Review of Scientific Instruments 84, 103710 (2013).

(16) L. Piazza, et al., Nature Communications 6, 6407 (2015).

(17) Materials, methods are available as supplementary materials. .

(18) A. Gliserin, M. Walbran, F. Krausz, P. Baum, Nature Communications 6, 8723 (2015).

(19) C. Kealhofer, et al., Science 352, 429 (2016).

(20) A. Lassise, P. H. A. Mutsaers, O. J. Luiten, Review of Scientific Instruments 83, 043705 (2012). 
(21) M. T. Hassan, H. Liu, J. S. Baskin, A. H. Zewail, Proceedings of the National Academy of Sciences of the United States of America 112, 12944 (2015).

(22) L. J. Wong, B. Freelon, T. Rohwer, N. Gedik, S. G. Johnson, New Journal of Physics 17, 013051 (2015).

(23) N. I. Zheludev, Science 328, 582 (2010).

(24) A. V. Kildishev, A. Boltasseva, V. M. Shalaev, Science 339, 1232009 (2013).

(25) R. A. Shelby, D. R. Smith, S. Schultz, Science 292, 77 (2001).

(26) X. J. Ni, Z. J. Wong, M. Mrejen, Y. Wang, X. Zhang, Science 349, 1310 (2015).

(27) D. Ehberger, et al., Physical Review Letters 114, 227601 (2015).

(28) P. Baum, A. H. Zewail, Proceedings of the National Academy of Sciences of the United States of America 104, 18409 (2007).

(29) A. Feist, et al., Nature 521, 200 (2015).

(30) W. Schneider, A. Ryabov, Cs. Lombosi, T. Metzger, Zs. Major, J. A. Fülöp, P. Baum, Optics Letters 39, 6604 (2014).

(31) L. Kasmi, D. Kreier, M. Bradler, E. Riedle, P. Baum, New Journal of Physics 17, 033008 (2015).

(32) M. V. Tsarev, D. Ehberger, P. Baum, Applied Physics B 122, 30 (2016).

(33) S. Lahme, C. Kealhofer, F. Krausz, P. Baum, Structural Dynamics 1, 034303 (2014).

(34) A. Lubk, J. Zweck, Physical Review A 91, 023805 (2015).

(35) R. Close, Z. Chen, N. Shibata, and S. D. Findlay, Ultramicroscopy 159, 124-137 (2015).

(36) Matthias Lohr, Ralph Schregle, Michael Jetter, Clemens Waechter, Thomas Wunderer, Ferdinand Scholz, Josef Zweck, Ultramicroscopy 117, 7-14 (2012).

(37) Reiner Bormann, Stefanie Strauch, Sascha Schaefer, Claus Ropers, Journal of Applied Physics 118, 173105 (2015). 
This work was supported by ERC and Munich-Centre for Advanced Photonics. We thank F. Krausz for support and inspiring discussions, and Ivan Zhuravlev (Vocord SoftLab) for help with programming. 
Fig. 1 Concept and experimental setup. A femtosecond laser produces single-cycle $\mathrm{THz}$ pulses (red) and a beam of femtosecond electron pulses (blue). The THz radiation compresses the electrons in time and also triggers electromagnetic resonance in the sample (yellow). The electron pulses are locally and instantaneously distorted (dotted lines) and therefore reveal the electrodynamics of the sample.

Fig. 2 Waveform microscopy results. (A) Electric field for sample excitation. (B) Electron pulse characterization (19). (C) Shape of the split-ring resonator. (D) Isosurface of the time-dependent shadow images. (E) Subset of raw images on the screen; see also movies S1 and S2.

Fig. 3 Space-time-field results in a split-ring resonator. (A) Time-frozen electric field vectors at three delay times; see movie S3. Scale bar, $100 \mu \mathrm{m}$. (B) Time-dependent fields at locations (1) and (2) in Fig. 3A. Right panels, polarizations. (C) Space-time map of the surface carrier density at the inner edge (white dashed line in Fig. 3A).

Fig. 4 Rectangle and butterfly resonators. (A) Time-frozen electric field vectors at $2.2 \mathrm{ps}$ delay; see movie S4. (B) Time-dependent field at the center. (C) First four modes revealed by singular value decomposition. (D) Time traces (amplitudes, scaled) of modes 1, 2 and 3. (E) Electric field strengths along a line through the center. (F) Butterfly resonator, electric field vectors at $2.8 \mathrm{ps}$ delay; see movie S5. Scale bars, $100 \mu \mathrm{m}$.

Supplementary Materials:

Materials and Methods

Figures S1-S4

Movies S1-S5

References (30-35) 


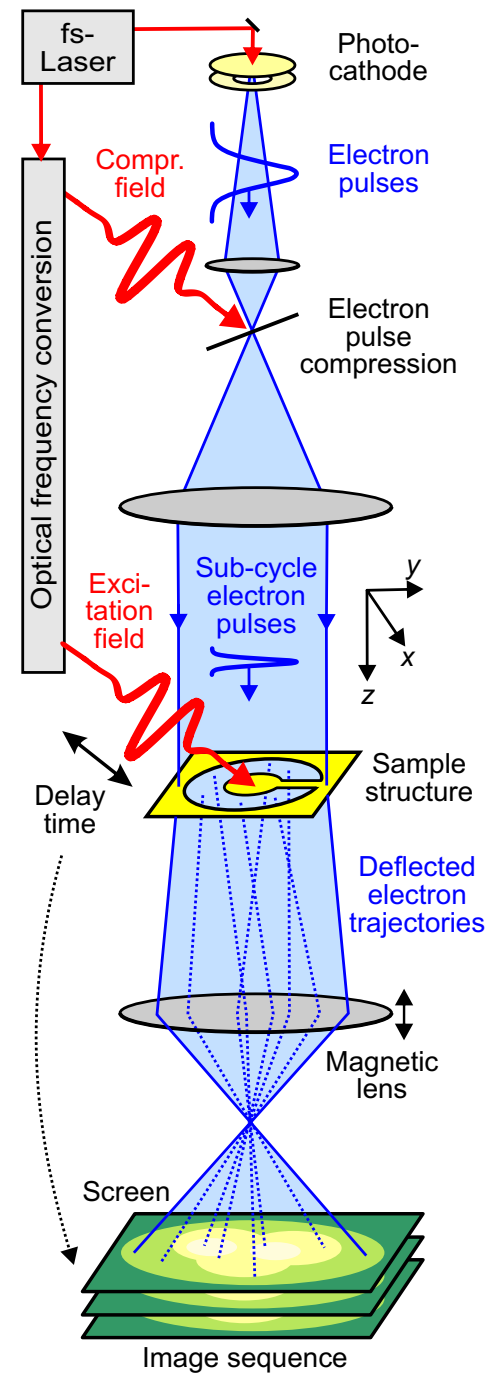



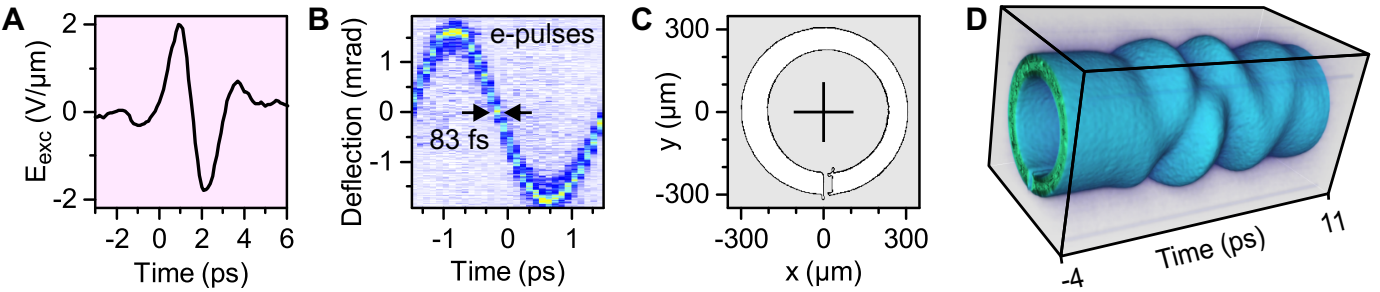

$E$

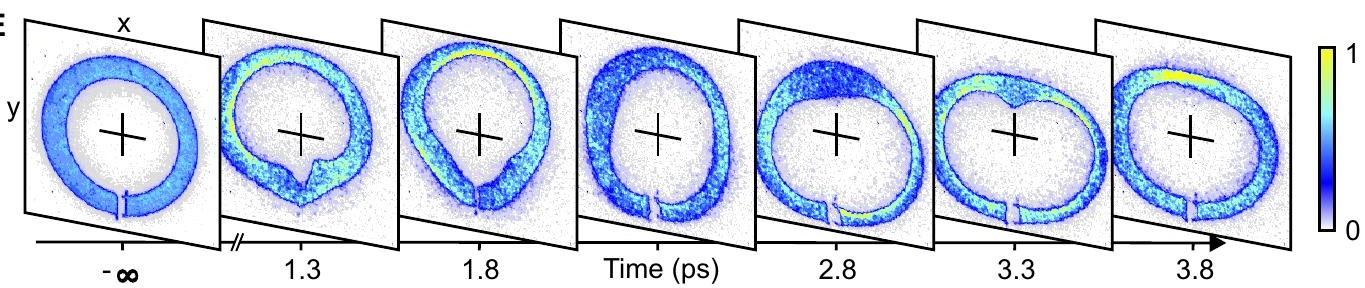




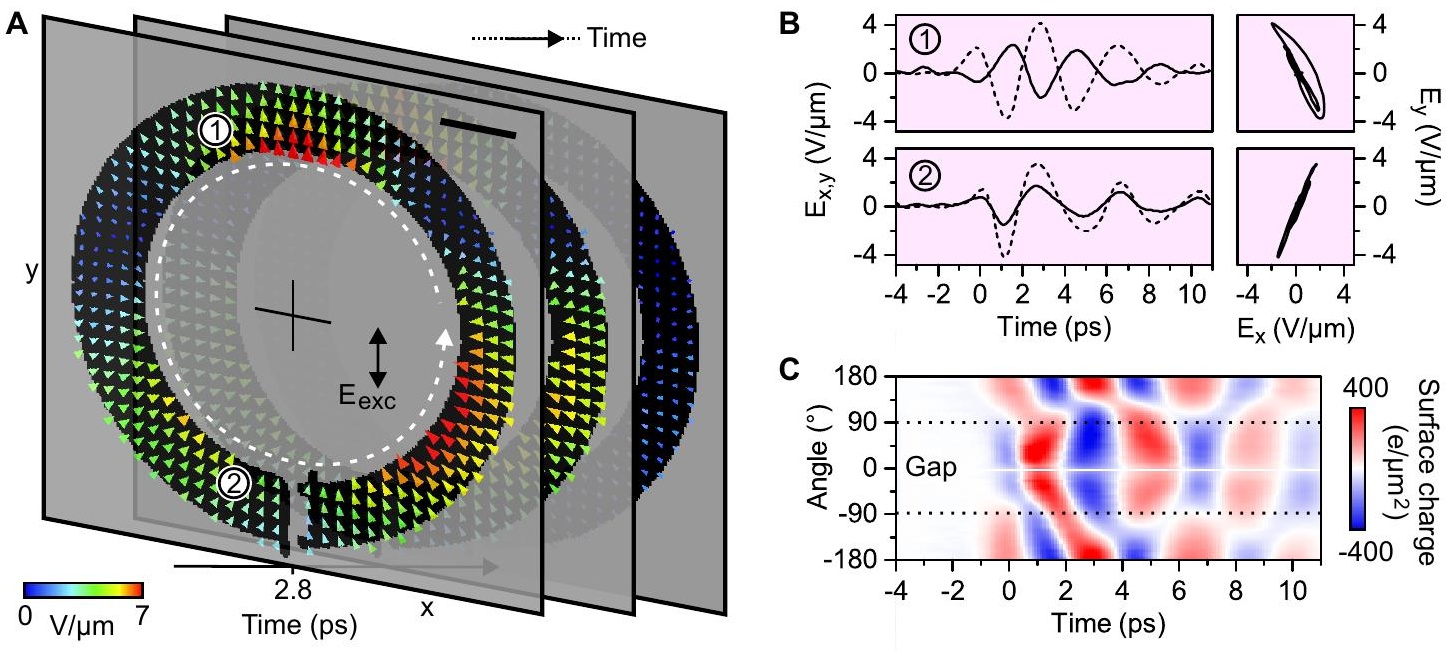




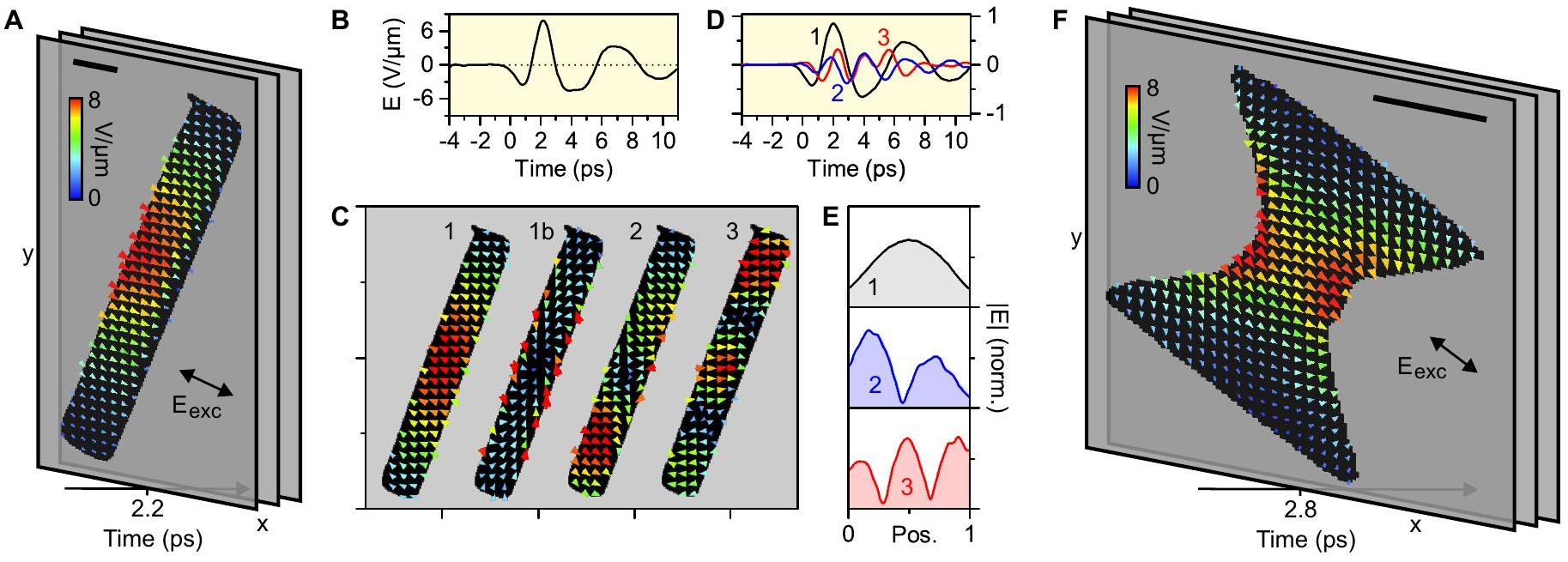




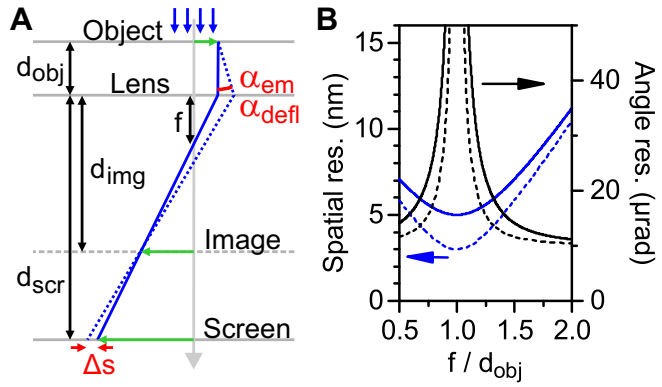




\section{Materials and Methods}

Experimental setup: The Yb:YAG disk laser (30) delivers 320-340 $\mu \mathrm{J}, \sim 1$ ps pulses at a repetition rate of $50 \mathrm{kHz}$ and at a central wavelength of $1030 \mathrm{~nm}$. A minor part of the output is frequency-doubled and triggers photoelectron emission from a 20-nm gold layer on a sapphire substrate via a two-photon process (31). We generate about ten electrons per pulse, accelerated to $70 \mathrm{keV}$ central energy, from an effective source size of $\sim 15 \mu \mathrm{m}$ (root-mean-square). A first magnetic solenoid lens, positioned $14 \mathrm{~cm}$ after the source's anode, focuses the electrons onto the THz-driven (32) electron pulse compressor (19). A second magnetic lens, located $9.5 \mathrm{~cm}$ after the compressor, produces a collimated beam. Its size was made big enough to illuminate the entire resonator structures with sufficient intensity. Waist scans and knife-edge measurements provide the beam emittance, virtual source size and divergence. The duration of the compressed electron

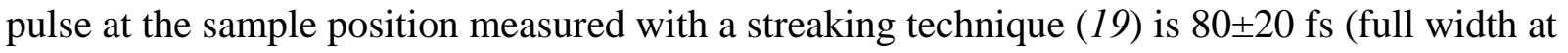
half maximum). The terahertz field for excitation is generated with tilted pulses in $\mathrm{LiNbO}_{3}(30)$ and focused onto the sample via an $f=51 \mathrm{~mm}$ off-axis parabolic mirror with a 3-mm-diameter hole for letting the electron beam pass through. All investigated samples are laser-drilled into 30$\mu \mathrm{m}$-thick aluminum foil. A third solenoid lens, placed $28 \mathrm{~cm}$ after the sample, images the shadow patterns on a phosphor screen coupled to a CMOS camera (TemCam-F416, TVIPS GmbH). The sample-detector distance is $134 \mathrm{~cm}$. The spatial resolution of this rudimentary microscope, based on an ultrafast single-electron diffraction beam line (34) with optimized temporal resolution (19), is $\sim 3 \mu \mathrm{m}$ at the low magnifications used for the resonator characterizations.
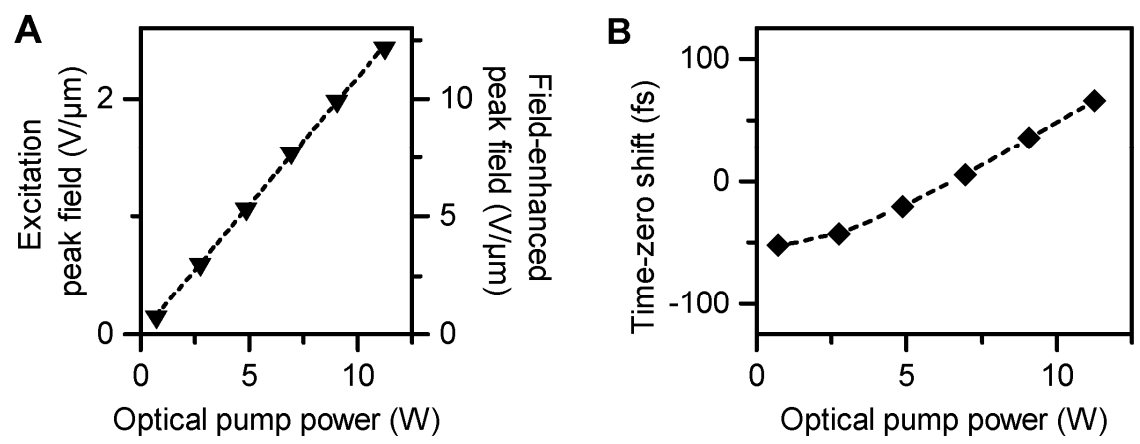

Fig. S1 Electric field and time-zero calibration. (A) Electric peak field strength versus optical pump power used for THz generation. The left scale denotes the field strength exciting the sample and the right scale that in the butterfly resonator's center. (B) Time-zero drift in dependence of the optical pump power, probably from temperature effects.

We vary the excitation field strength with a thin-film polarizer and motorized half-wave plate in the optical pump beam driving the terahertz generation. Figure S1A demonstrates the linearity of this approach. Some correlated shifts of time-zero in the infrared beamline were characterized by 
streaking (see Fig. S1B) and compensated optomechanically in the field-strength scans. A maximum optical pump power of $8.6 \mathrm{~W}$ and thereby a maximum incident field of $\sim 2 \mathrm{~V} / \mu \mathrm{m}$ was applied in the experiments. The average $\mathrm{THz}$ power on the sample is $\sim 1.2 \mathrm{~mW}$ in a mm-sized focus. Accordingly, there are no temperature effects in the sample. The electron beam, at only $\sim 10$ e per pulse, also causes no heating or charging effects.
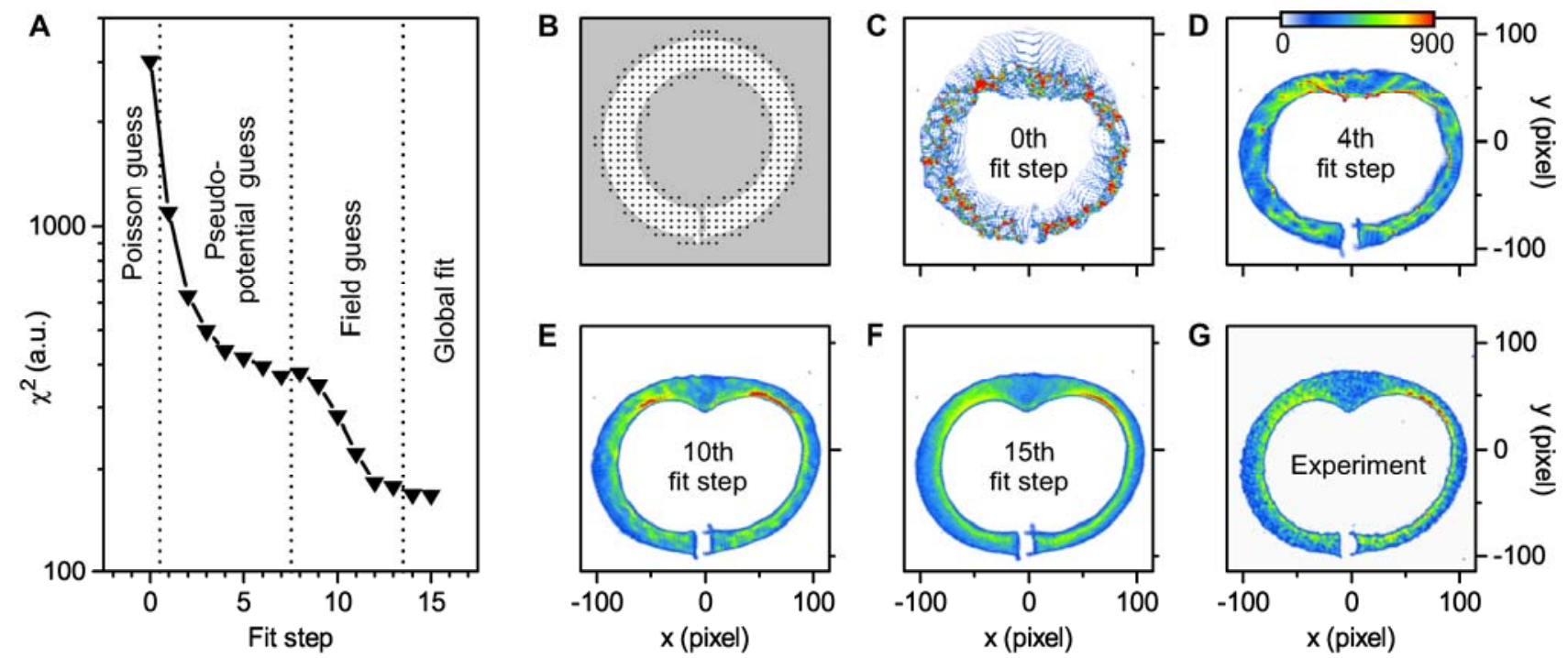

Fig. S2 Fit convergence and grid. (A) Evolution of squared differences $\chi^{2}$ between fit and data batch though the three stages of guessing and final fit. The $14^{\text {th }}$ and $15^{\text {th }}$ fit steps show $\chi^{2}$ after 15 and 31 iterations of global fit, respectively. (B) Assignment of spline points on the split-ring mask. $(C)-(F)$ Evolution of the fitted highest-excitation images through the guessing and fitting procedure. $(G)$ Measured highest-excitation image.

Data analysis: At every pump-probe delay time $\tau$ there is a batch of 16 images at varying excitation field strength and one mask image $\left(0^{\text {th }}\right)$, taken without the field. For calculating the sample's map $\alpha_{x, y}(x, y, \tau)$ of deflection angles for each batch and for each delay step, a leastsquare fitting algorithm (Gauss-Newton) is invoked. We parameterize $\alpha_{x}(x, y, \tau)$ and $\alpha_{y}(x, y, \tau)$ with $2 \times \mathrm{N}$ spline support points over the sample's open area, where $\mathrm{N}=405$ (see Fig. S2B). A fit optimizes $\alpha_{x, y}(x, y, \tau)$ by minimizing the difference between experimentally acquired images and a trajectory projection, which is obtained by oversampling the incoming electron beam 7-fold via a 2D bi-harmonic spline function. We propagate the incoming electron density derived from the mask image to the $i$-th image on the screen as straight particle trajectories, bent by $\alpha_{x}$ and $\alpha_{y}$, and subsequently optimize the spline parameter values by minimizing the difference to the 16 experimental images with a Gauss-Newton algorithm. Spline function, propagation and the minimization are computed with a 3072-threaded graphics processing unit (GeForce GTX Titan X, NVIDIA Corporation). 
We apply three guessing steps and a final global fit. As a first guess we solve the Poisson equation for the difference between the mask image and the $1^{\text {st }}$ image in the batch, in which deflection is the lowest. The idea is that locally convergent or divergent beams correspond to more or less intensity on the screen, respectively (34). As a result we have a 2D pseudo-potential distribution describing the deflection.

A second, better guess is obtained by least-square fitting the 2D pseudo-potential for a series of image pairs, i.e. $0^{\text {th }}$ and $2^{\text {nd }}$, then $0^{\text {th }}$ and $3^{\text {rd }}$, up to $0^{\text {th }}$ and $8^{\text {th }}$, while each time using the result as a new guess. This is a converging series, because the low-order images are more prominently revealing the stronger field components, while the higher-order images become increasingly more sensitive to the weaker fields.

In the third guessing step, we drop the assumption of a 2D pseudo-potential and now allow two independent deflection components, i.e. $\alpha_{x}(x, y, \tau)$ and $\alpha_{y}(x, y, \tau)$, thereby doubling the amount of fit parameters. Like before, we subsequently fit image pairs, namely $0^{\text {th }}$ and $6^{\text {th }}, 7^{\text {th }}, 9^{\text {th }}$, $12^{\text {th }}, 14^{\text {th }}, 16^{\text {th }}$, respectively.

For the final fit, the complete batch of images (1-16) is used for a global fit of $\alpha_{x}(x, y, \tau)$ and $\alpha_{y}(x, y, \tau)$. This procedure takes about 3 minutes for the 810 spline points and $230 \times 230$ pixel $^{2}$ images of the split-ring resonator data. $E_{x}(x, y, \tau)$ and $E_{y}(x, y, \tau)$ are computed via Eq. (1) and the magnification. The time-resolved results are obtained by automatically repeating the whole procedure for all time steps independently. Because the problem is 2500 times overdetermined, the fit converges well and more complex inversion procedures $(34,35)$ are not required. All time steps are fitted independently. Figure S2A depicts the fit convergence $\chi^{2}$ of the batch at 3.1 ps delay. The small increase after allowing free fields is due to reassigning the spline points. Figures S2C-F show the gradual convergence of the highest-excitation image to the experimental data (Fig. S2G). Different variations of the Poisson guess produce the same final results. We also tested the algorithm with simulated data (CST Microwave Studio and PIC Solver, CST GmbH), confirming its correct convergence. The simulations also confirm the general shape of the measured vector fields reported in Figs. 3-4.

Nonlinear effects: Investigating nonlinear or strong-field phenomena does not permit varying the excitation field strength as here applied for solving the data inversion problem. In case that nonlinear optical effects are expected, the image series required for data inversion at strong deflections can alternatively be achieved at constant field strength with a series of defocus conditions. 
Magnetic fields: Numerical simulations (CST Microwave Studio, CST GmbH) validate that magnetic fields can be neglected in a first approximation. Figure S3 shows as an example the magnetic field in the split-ring resonator's gap area. The peak field is $28 \mathrm{mT}$, but the field vectors are oriented within the structure mostly in parallel to the electron beam direction $(\mathrm{z})$ and are mostly anti-parallel above and below.

First, the interaction of the beam with the longitudinal magnetic fields is determined by the residual sideways components $\alpha_{e m} v_{e}$ in the electron trajectories. The ratio $C_{\|}$of longitudinalmagnetic to sideways-electric deflection can be estimated as $C_{\|} \approx\left|\int \alpha_{e m} v_{e} B_{z} d z\right| /\left|\int \boldsymbol{E}_{\perp} d z\right|$. For all positions and pump-probe delays, $C_{\|}$is less than $10^{-3}$ in the simulations. Deflection effects of longitudinal magnetic fields are therefore negligible. Second, the effect of sideways magnetic fields above and below the resonator plane is largely cancelled for electron trajectories along $\mathrm{z}$.

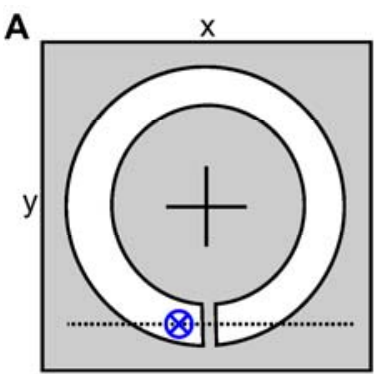

Electrons
B

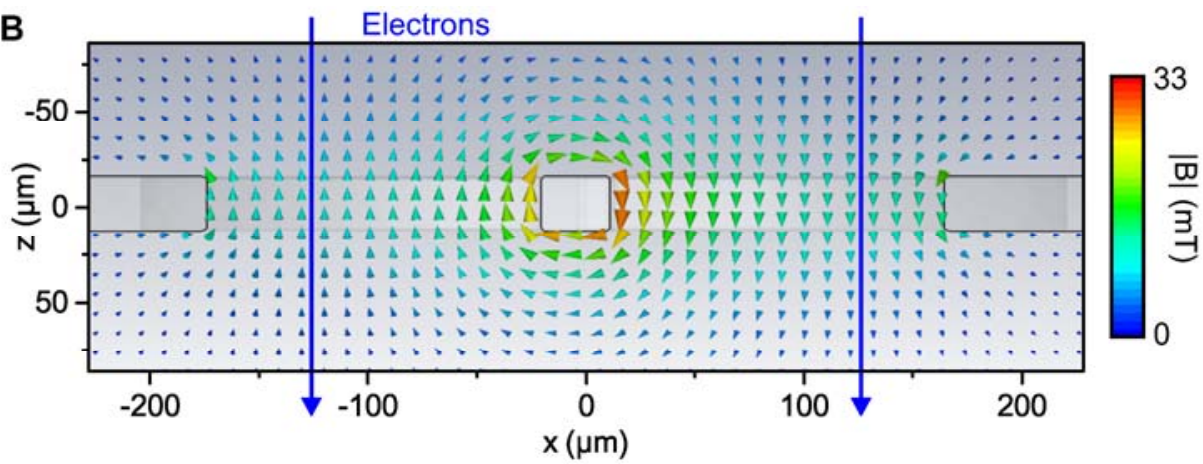

Fig. S3 Simulated magnetic fields. (A) Sketch of the split-ring resonator with the electron probing direction marked in blue. (B) Magnetic field vector distribution in the plane marked in (A) with the dotted line at 3.1 ps delay. Electron trajectories are marked in blue.

In the simulations, the ratio $C_{\perp}=\left|\int v_{e} \boldsymbol{B}_{\perp} d z\right| /\left|\int \boldsymbol{E}_{\perp} d z\right|$ is typically $\sim 2 \%$ in all regions producing reasonable net deflection, and play therefore a minor role. In principle, transverse magnetic components could be measured by comparing waveform electron microscopy experiments at different electron velocities.

Resolution estimations: While our experimental arrangement (Fig. 1) has provided the necessary temporal resolution for entering the regime of sub-cycle imaging, the apparatus has not been specifically designed for imaging quality. What are in principle the limits of waveform electron microscopy in spatial and angular resolution? With a continuous electron beam, 5-nm and 2- $\mu$ rad resolution have been achieved simultaneously in a scanning geometry (36). For our case of collimated sub-cycle electron pulses and imaging with defocus, we invoke a ray-optical picture as a zeroth-order approximation (Fig. S4A). The object and screen positions $d_{o b j}$ and $d_{s c r}$ are fixed, while the image plane location $d_{i m g}$ is defined by the lens strength $f$ via $1 / f \approx 1 / d_{\text {obj }}+1 / d_{\text {img }}$. The magnifications (green arrows) at image and screen are $M_{i m g} \approx d_{i m g} / d_{o b j}$ and $M_{s c r} \approx$ $M_{i m g}\left(d_{s c r}-f\right) /\left(d_{i m g}-f\right)$, respectively. We assume that the microscope has a finite, instrument-limited resolution $R_{0}$ which produces a minimum measurable feature size $M_{s c r} R_{0}$ at 
the screen. The illuminating wide beam (emittance $\varepsilon$ and radius $R$ ) has at each sample location an angular spread of $\alpha_{e m}=\varepsilon / R$ (dotted line). This causes a feature size increase of $\Delta \mathrm{s} \approx$ $\left|M_{i m g}{ }^{-1} \alpha_{e m}\left(d_{s c r}-d_{i m g}\right)\right|$ at the screen. The spatial resolution $R_{x}$ at the sample therefore degrades from $R_{0}$ approximately via $R_{x}{ }^{2} \approx\left(\Delta \mathrm{s} / M_{s c r}\right)^{2}+R_{0}{ }^{2}$. For the angular resolution $R_{\alpha}$, we assume that a sample-induced deflection $\alpha_{\text {defl }}$ is well discernible if the displacement it causes on the screen exceeds the resolution-limited feature size $M_{s c r} R_{x}$. We obtain $R_{\alpha} \approx \mid M_{i m g} /\left(d_{i m g}-\right.$ $\left.d_{s c r}\right) \mid M_{s c r} R_{x}$. A more detailed analysis requires numerical approaches $(34,35)$. For obtaining Fig. $\mathrm{S} 4 \mathrm{~B}$, we assumed values realistic in femtosecond electron microscopy $(1,27,37)$, namely an emittance of $20 \mathrm{pm}$, which is ten times worse than achievable $(27,37)$, an illuminated spot of $2 \mu \mathrm{m}$ radius, a magnification of $\sim 10,000$ and an effective microscope resolution limit of 3 or $5 \mathrm{~nm}$ (38). While at perfect imaging ( $f \approx d_{o b j}$ for large magnification) the angular resolution diverges, it improves at increasing defocus; the limit is $\alpha_{e m}$.

Tens of $\mu$ rad are sufficient to measure the fields in computer circuitry, where a typical operating voltage is $\sim 1 \mathrm{~V}$ and the structures are tens of $\mathrm{nm}$ in size. For plasmonics, the field strengths vary a lot, but for example tens of $\mu \mathrm{W}$ of optical power in a tens-of-nanometer-diameter photonic waveguide corresponds to $\mu$ rad deflections.

The experiment's angular resolution can be estimated by analyzing the noise of the time traces (e.g. Figs. 3B and 4B) without the 1-THz low-pass filter. We find a standard deviation of $\sim 0.3 \mathrm{~V} / \mu \mathrm{m}$ and $\sim 0.4 \mathrm{~V} / \mu \mathrm{m}$, respectively, which corresponds via Eq. (1) to 60-80 $\mu$ rad resolution in our experiments. This measured value is close to $\alpha_{e m} \approx 60 \mu \mathrm{rad}$, the divergence of the illuminating beam, and therefore corroborates the above considerations of the achievable resolutions.
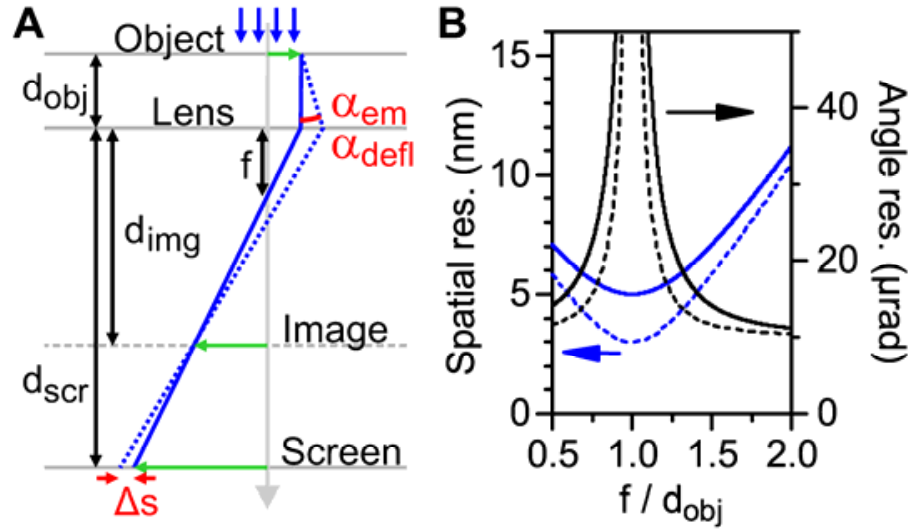

Fig. S4 Estimated resolution limits of waveform electron microscopy. (A) Geometrical optical picture. An object is illuminated by a wide electron beam (blue arrows) with an intrinsic divergence $\left(\alpha_{e m}\right)$. Local time-frozen fields cause an additional deflection of the electron trajectory $\left(\alpha_{\text {defl }}\right)$. In case of defocus, features widen on the screen by $\Delta s$. (B) Estimated spatial (black) and angular (blue) resolution of waveform microscopy in dependence of defocus $\left(f / d_{o b j}\right)$ for a realistic femtosecond microscope with $20 \mathrm{pm}$ beam emittance and $3 \mathrm{~nm}$ (dotted) or $5 \mathrm{~nm}$ (solid) basic spatial resolution. 20 rad deflection is sufficient to measure the electric fields in high-speed electronics. 
Movie S1 Time-dependent raw images of the split-ring resonator. The displayed screen area is $3.5 \times 3.5 \mathrm{~mm}^{2}$ and the color scale is the same as in Fig. 2. Each pump-probe image is integrated for $3 \mathrm{~s}$ with single-electron pulses at $50 \mathrm{kHz}$.

Movie S2 Time-dependent raw images of the butterfly resonator. The displayed screen area is $3.5 \times 3.5 \mathrm{~mm}^{2}$ and the color scale is the same as in Fig. 2 .

Movie S3 Time-dependent field vectors in the split-ring resonator. The image size is $660 \times 660 \mu^{2}$ and the color scale is the same as in Fig. 3A. The triangle directions denote where the field vectors point to.

Movie S4 Time-dependent field vectors in the rectangular slit. The image size is $400 \times 800$ $\mu^{2}$ and the color scale is the same as in Fig. $4 \mathrm{~A}$.

Movie S5 Time-dependent field vectors in the butterfly resonator. The image size is $460 \times 460 \mu^{2}$ and the color scale is the same as in Fig. $4 \mathrm{~F}$. 